\title{
User-Centric and Intelligent Service Composition in Ubiquitous Computing Environments
}

\author{
In-Young Ko \\ Computer Science Department, \\ Korea Advanced Institute of Science and Technology (KAIST) \\ Daejeon, Korea \\ iko@kaist.ac.kr
}

\begin{abstract}
The advancement of service-oriented computing and mobile device technologies gives us new challenges to provide intelligent services in ubiquitous computing (ubicomp) environments. User-centricity and dynamism support are the most essential requirements to meet those challenges. In this talk, I will introduce a user-centric and intelligent service composition framework that allows users to create their personalized ubicomp applications that utilize service resources in a highly dynamic ubicomp environments. The main features of our framework include: (1) task-oriented and spontaneous service composition; (2) dynamic service monitoring and reconfiguration; and (3) pervasive service retrieval and management. I will also explain our experiences of applying this framework to urban computing applications and intelligent service robots.
\end{abstract}

